\title{
A Method for Data Extraction from Video Sequences for Automatic Identification of Football Players Based on Their Numbers
}

\author{
Dariusz Frejlichowski \\ West Pomeranian University of Technology, Szczecin, \\ Faculty of Computer Science and Information Technology, \\ Zolnierska 52, 71-210, Szczecin, Poland \\ dfrejlichowski@wi.zut.edu.pl
}

\begin{abstract}
In the paper the first stage of the approach for automatic identification of football players is presented. It is based on the numbers placed on their shirts. The method works with video frames extracted during a television sport broadcast. The element of the system described in this paper is devoted to the localisation of the numbers and their extraction for future recognition. It is simple, yet efficient and it is based on the use of appropriate ranges in various colour spaces. Four colour spaces were experimentally evaluated for this purpose. Thanks to this, the ranges could be established for particular kits. Firstly, the part of an image with a shirt was localised, and later, within this area, a number was found.
\end{abstract}

Keywords: players identification, feature localisation, colour spaces.

\section{Introduction}

Automatic identification of football players (or other sport disciplines) during a TV broadcast is a difficult and challenging task. However, the possible benefits arising from the use of a system realising such a function, especially taking into account the popularity of this sport, are providing researchers with constant work in this field. The aim is the identification of a sportsman (in real time) during a TV transmission, performed automatically, without (or in small level, semi-automatically) human interaction.

Many approaches, and even complete systems have been proposed so far (e.g. MATRIS [1], TRACAB [2], MELISA [3]), but in many cases they are not completely automatic or are limited to the problem of tracking. The most common approach is the use of a human operator of the broadcast, supported by a computer application. Usually a computer is only used to make the broadcast more attractive. In case of automatic systems employing image analysis and recognition algorithms and based on video sequence (or single frames extracted from it), two approaches are especially interesting. The first one is more popular and has 
been explored for a longer period of time. It is based on the extraction and identification of numbers placed on the back of players' shirts. The second approach makes use of the most popular biometric feature - the face.

An example of the first approach is a method presented in [4], where the tracking of players and identification based on numbers was performed. The method used watersheds and Region Adjacency Graphs for feature localisation and extraction, and n-tuple, Hidden Markov Models and Neural Networks for classification. In [5] a simple descriptor was used for finding a white region on red-black stripes. The candidate regions were merged by means of graphs. The extracted numbers were identified with the use of the Principal Component Analysis (PCA).

An example of the second approach was presented in [6]. The identification described there was based on the players' faces. Firstly, scene change detection was carried out by means of the image histogram. Secondly, close-up detection was performed, which made the appearance of a face in a scene more probable. The next stage was the face localisation through searching for skin colour pixels in $\mathrm{YCbCr}$ and HSV spaces, followed by template matching. The identification stage involved Discrete Fourier Transform for feature extraction and PCA+LDA (Linear Discriminant Analysis) for feature reduction. This approach is strongly similar to [7], where some general biometric problems were explored and solved.

Sometimes, only the location of players is explored, e.g. for that purpose in 8 so-called mosaic images were used, and in 9] approximation of players' location (points in image) according to the play field model.

It is worth to mention that not only players are localised (and sometimes tracked or identified). In 10] particular events (e.g. shot on goal, penalty, free kick) were detected, basing on fast camera movement (with the use of MPEG vectors). Similarly, in [11] 'highlights' were detected with the use of template matching, finding of play field zones and Finite State Machines. In [12] only the ball was detected by means of Support Vector Machines. Unfortunately, this approach was developed for traditional black and white football, therefore it is less practical nowadays.

The decision to grant the hosting of the 2012 UEFA European Football Championship to Poland and Ukraine has resulted in the significant increase of interest in those countries in the automatic analysis of video sequences coming from football matches. Identification of players in order to make the broadcast more attractive is the first obvious application. However, some other areas can be easily pointed out, e.g. analysis of the crowd connected to required standards of safety and security measures or an automatic analysis of exposure or visibility time of the advertising banners.

In this paper a method for automatic localisation of football players' kits and numbers is presented. It is the first stage of the algorithm for automatic identification of players basing on their numbers. The approach was tested on video sequences recorded during the World Cup in Germany in 2006 and partially presented in 13. In the previous publication the entire proposed approach was outlined, but emphasis was placed on the localisation of shirts. However, it only 
constitutes the first part of the process. Since the goal is the automatic identification of players, the subsequent stage is more important, namely the extraction of the numbers placed on them. Hence, it constitutes the main topic of this paper - the approach and experimental results for this stage are presented. The extraction of a number from a shirt is described in Section 3. Earlier, in Section 2 the localisation of a shirt is briefly recalled.

\section{Localisation of Players Based on Information about Their Kits Colour}

The first step in the proposed approach is the localisation of pixels in a frame that belongs to a player on the play field. It is carried out by using information about the colours of sport kits assigned to teams. It is known that according to certain sports laws the kits of the two playing teams (and referees) have to be clearly different. Therefore, all football teams (not only national) have to use two (so called "home" and "away" colours), and sometimes even three kits. After the localisation of a shirt we are looking for numbers placed on it. In both cases (shirts and numbers) the appropriate ranges of values in colour spaces were experimentally established. Such an approach was applied with success in face localisation based on the skin colour ([7]).

A football player's kit is composed of three elements: a shirt, shorts (goalkeepers are allowed to wear tracksuit bottoms instead) and socks. In the proposed approach the first from the listed elements is especially important, because it constitutes the largest part of a kit and, what is more important, the numbers to be recognised are placed on it. However, if the colour of shorts and socks is the same as the colour of the shirts, they will be localised as well. It is not a problem, because the only difference is the larger area to explore in the next stage.

Four colour models were tested, namely: RGB, HSV, YCbCr and CIE L*a*b. Amongst them the best results were obtained when using $\mathrm{YCbCr}$ and $\mathrm{HSV}$. It was caused by the characteristics of these colour spaces. Most important is the presence of a component more general from the other in particular model, e.g. Hue in HSV and luma in YUV. In the second case blue-difference and reddifference chroma components were even more helpful.

The process of establishing the appropriate ranges within the particular colour model components was based on the exploration of several dozens of shots for each colour of a kit (this number was different and depended on the 'uniqueness' of a colour) taken from various football matches. In some cases the localisation was successful in various spaces (for example the yellow colour was easy to extract in each explored model). If so, the one which separated a shirt from the rest of an image best was chosen. The achieved ranges for each colour class appearing during the World Cup 2006 are provided in Table 1]([13]). For the purpose of the experiments the image sequences recorded from analogue TV during the FIFA World Cup 2006 in Germany (AVI, $720 \times 576$, interlaced video, MPEG-2) have been used. Some examples are provided in Fig. 1 . 

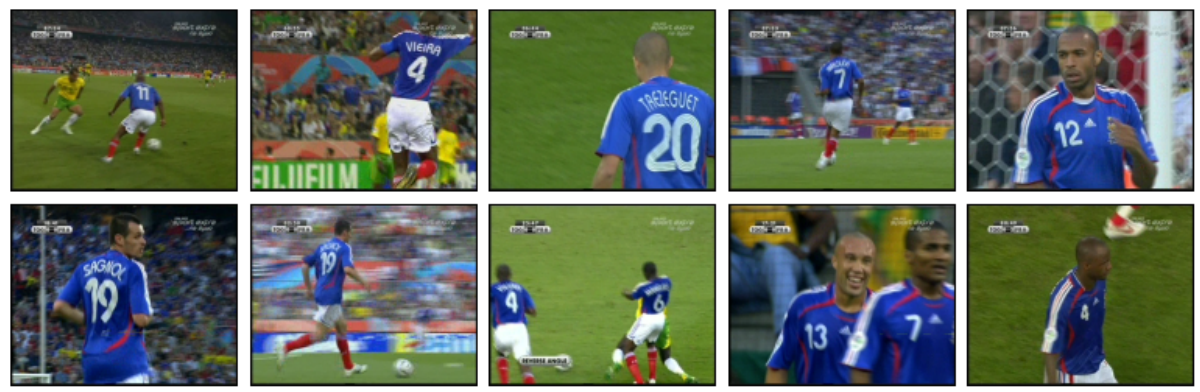

Fig. 1. Examples of the experimental data - frames for one class (white numbers on blue shirts, 13]

Table 1. The experimentally established ranges within the colour models for the localisation of players' shirts

\begin{tabular}{|c|c|c|c|c|c|c|c|c|c|c|}
\hline & white & red & yellow & $\begin{array}{l}\text { white- } \\
\text { red }\end{array}$ & $\begin{array}{l}\text { white- } \\
\text { blue }\end{array}$ & orange & green & blue & maroo & $\begin{array}{l}\text { navy- } \\
\text { blue }\end{array}$ \\
\hline$\overline{\mathrm{Y}}$ & $>90$ & - & - & $\begin{array}{l}<85 \text { or } \\
>140\end{array}$ & - & - & - & - & $<85$ & - \\
\hline $\mathrm{Cb}$ & $\begin{array}{l}120- \\
150\end{array}$ & $90-130$ & $30-80$ & $90-150$ & $\begin{array}{l}120- \\
145\end{array}$ & - & $95-120$ & $\begin{array}{l}135- \\
190\end{array}$ & $\begin{array}{l}115- \\
135\end{array}$ & - \\
\hline $\mathrm{Cr}$ & - & $>145$ & $\begin{array}{l}130- \\
170\end{array}$ & $\begin{array}{l}<125 \\
\text { or } \\
>160\end{array}$ & $\begin{array}{l}110- \\
135\end{array}$ & - & $\begin{array}{l}100- \\
122\end{array}$ & $95-125$ & $\begin{array}{l}135- \\
165\end{array}$ & - \\
\hline $\mathrm{H}$ & - & - & - & - & - & $\begin{array}{l}0.045- \\
0.1\end{array}$ & - & - & - & $0.4-0.7$ \\
\hline $\mathrm{S}$ & - & - & - & - & - & $>0.7$ & - & - & - & $\begin{array}{l}0.22- \\
0.8\end{array}$ \\
\hline V & - & - & - & - & - & - & - & - & - & - \\
\hline
\end{tabular}

In the achieved image we have to reject some small undesirable objects that can appear as a result of various image or video distortions. In the proposed approach this was performed on the basis of the analysis of the boundary length of an object. The performed experiments gave the appropriate threshold - the length of the boundary has to exceed 200 points.

Unfortunately, in some cases the abovementioned method did not prove successful. This concerns the situations in which some other visible objects with the same colour as the requested shirt (e.g. banners) can be extracted as well (see Fig. 11). Hence, in order to remove them, it was assumed (basing on the experiments) that the product of both sizes of a rectangle enclosing the candidate object has to be higher than 0.65 and lower than 1.75 ([13]). 

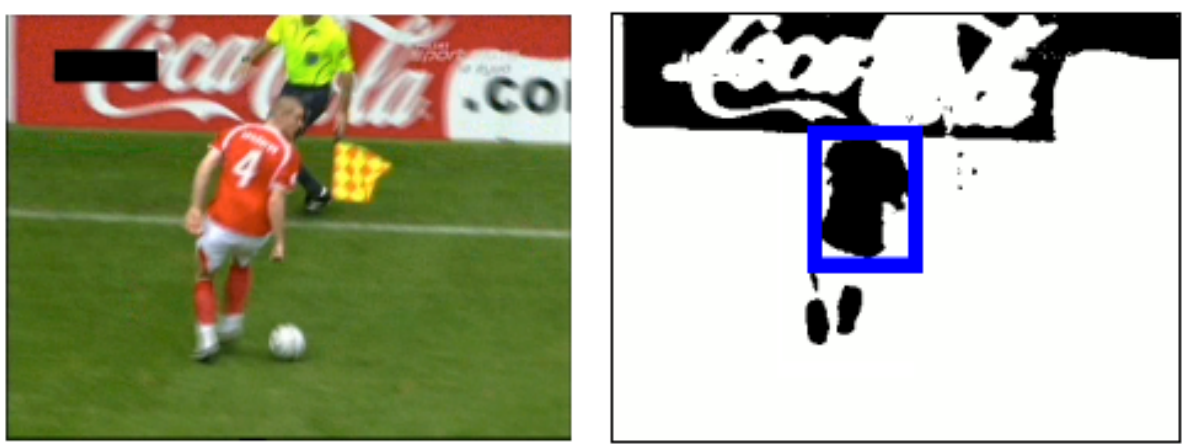

Fig. 2. The result of the localisation of a shirt

\section{Extraction of a Number Placed on a Shirt}

After performing the localisation of a shirt — the second problem concerned localisation of numbers placed on them. Unfortunately, the ranges established in the previous stage could not be applied again. This problem was mainly caused by the influence of the colour of the shirt on the colour of the number, e.g. blue digits enclosed by a red colour should be localised using other ranges than by white ones. Hence, new ranges in colour spaces were experimentally established. They are provided in Table $2([13])$.

Table 2. The experimentally established ranges within the colour models for the localisation of numbers within the previously extracted frame area belonging to a shirt

\begin{tabular}{lllllllll}
\hline $\begin{array}{l}\text { white } \\
\text { (red) }\end{array}$ & $\begin{array}{l}\text { white } \\
\text { (blue) }\end{array}$ & $\begin{array}{l}\text { white } \\
\text { (green) }\end{array}$ & yellow & $\begin{array}{l}\text { black } \\
\text { (blue- } \\
\text { white) }\end{array}$ & $\begin{array}{l}\text { black } \\
\text { (orange) }\end{array}$ & $\begin{array}{l}\text { green } \\
\text { blue } \\
\text { (white- } \\
\text { blue) }\end{array}$ & $\begin{array}{l}\text { blue } \\
\text { (white) }\end{array}$ \\
\hline $\mathrm{Y}>110$ & $>130$ & $>95$ & - & $45-130$ & $105-130$ & - & - & - \\
$\mathrm{Cb} \mathrm{125-155}$ & $125-160$ & $115-145$ & $65-105$ & $120-140$ & $125-175$ & $60-95$ & - & - \\
$\mathrm{Cr}-$ & - & - & $135-155$ & - & - & $110-140$ & - & - \\
$\mathrm{H}-$ & - & - & - & - & - & - & $0.45-0.6$ & $0.3-0.8$ \\
$\mathrm{~S}-$ & - & - & - & - & - & - & - & $0.22-$ \\
$\mathrm{V}-$ & - & - & - & - & - & - & $0.19-0.6$ & $0.3-0.75$ \\
\hline
\end{tabular}

The extracted numbers can be recognised with the help of shape descriptors. In case of international competitions employing national teams, such as The World Cup, The Confederations Cup, continental tournaments governed by particular football associations (e.g. The UEFA European Football Championship, The AFC Asian Cup, The Africa Cup of Nations, Copa America) usually the 

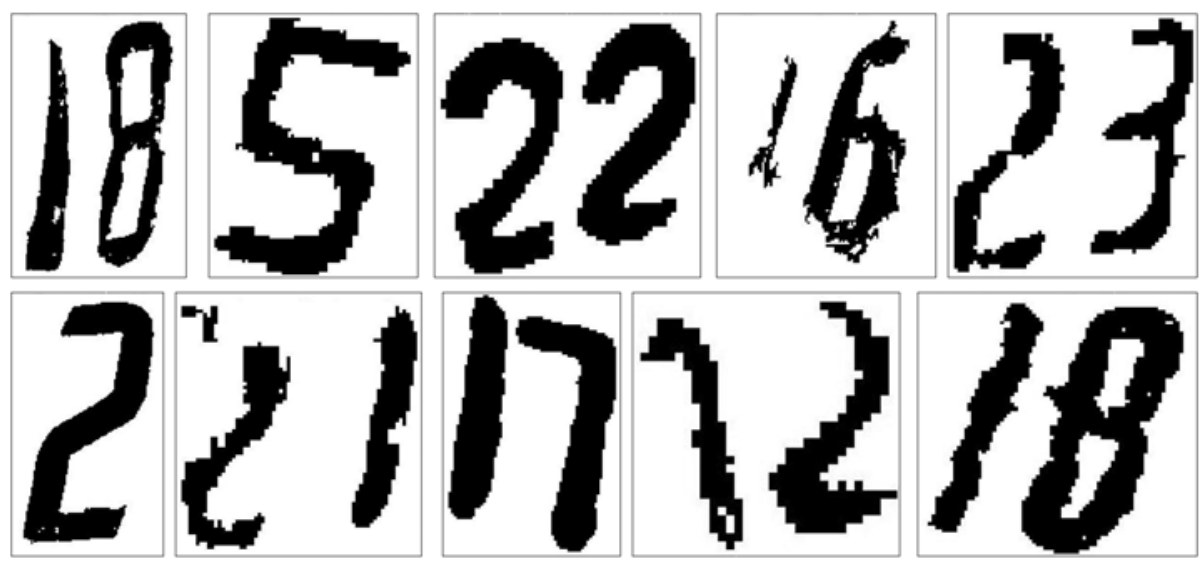

Fig. 3. Examples of numbers on sports shirts extracted by means of the approach described in the paper

problem of recognition of numbers can be made easier. It results from the strict regulations assumed, e.g. the limitations for possible numbers — from 1 to 23 . It is different than in the case of football clubs, where those numbers are more inconstant.

The recognition of particular numbers can be more facilitated when taking into consideration only the players present on the play field at the moment. That gives at the most eleven possible numbers for one team. Moreover, the whole numbers can be treated as shapes for recognition, not the single digits. The small number of template classes enables it. However, in this case we have to combine the digits for two-digit numbers. It is simple, because if there are two 'candidates for digits' close to each other we can assume that they can produce a number. In the experiment this task was carried out through calculating the centroids of localised objects within the area of a shirt. If they were close in vertical and horizontal directions and the 'candidates' were sufficiently large, they were merged.

The last step in the approach is the rejection of small localised objects. Usually those are some undesirable objects that should not be taken into consideration. On the other hand, if those objects are numbers and are small it is still less probable to perform successful recognition using them. Therefore, they can be rejected without any influence on the overall efficiency of the approach. Some examples of the extracted using the described approach numbers are provided in Fig. 2. As can be seen the shape of extracted numbers can be strongly distorted, e.g. by creases in the shirts or imaging and weather conditions. It will strongly hamper the later recognition of numbers.

In order to estimate the efficiency of the proposed approach the experiment was performed, using 20 video sequences, lasting from 2 to 3 minutes, from various matches played during the 2006 World Cup. For each of them single frames 
were extracted, 30 per minute. A frame was taken for the later processing if it contained at least one player with a visible number. An algorithm for the localisation of a shirt, described in Section 2, was applied for it. The frames with properly extracted shirts were subjected to the method of number localisation, presented in this section. Then, the obtained result was compared with the answers provided by humans. The obtained statistics — the percentage of artificial results accepted by the human operator are presented in Table 3.

Table 3. The experimental results of localisation of numbers obtained for particular colours of shirts

\begin{tabular}{lccccccccc}
\hline No. of frames & 52 & 63 & 31 & 67 & 47 & 19 & 29 & 38 & 49 \\
No. of correct results & 33 & 42 & 25 & 36 & 20 & 15 & 12 & 13 & 21 \\
Efficiency & $64 \%$ & $67 \%$ & $81 \%$ & $54 \%$ & $43 \%$ & $79 \%$ & $41 \%$ & $34 \%$ & $63 \%$ \\
\hline
\end{tabular}

The obtained results are far from ideal. The average efficiency was equal to $58 \%$ (227 from 395 investigated frames were properly processed). However, the very difficult nature of the investigated problem has to be emphasised. Apart from the mentioned strong distortions of the numbers there are many other problems to consider. Some of them were obvious even before starting the experiments. First of all, not every camera shot can be explored. For instance, it is useless to work with shots taken from long distance. In that case the player's number is not visible. Secondly, the grass's green colour is especially difficult in the method. During the World Cup 2006 three teams, namely Togo, Ireland and Ivory Coast, had green shirts. It is obvious that in some cases (e.g. depending on lighting conditions) the result of the extraction will cover a larger area, including some parts of the play field as well. The kit of a goalkeeper constitutes another problem. Usually it strongly differs from the original national kit. The same problem is related to a referee, but he does not lie in the area of interest as far as the proposed application is concerned. The very difficult problem is caused by some distortions of clothes, due to rain, dirt, tearing, etc. Those can significantly change the overall colour of a shirt.

Another interesting issue is the use of numbers strictly designed and prepared for a team. They are designed by the sports apparel manufacturers for particular national teams (sometimes more than one) and are used for some time (usually, at least one year) by them. This is helpful in the preparation of the templates for a particular football match.

\section{Conclusions}

In the paper an approach for the extraction of the numbers placed on sport shirts during the TV broadcast was proposed. This is the first step in the automatic system for identification of players basing on their numbers and is composed 
of two main stages: localisation of kits and later - numbers within the previously selected image area. The research was limited to the football matches. The experimentally established ranges in various colour spaces were used for the localisation.

Future works will be concentrated on the second stage - the recognition of the extracted numbers. Several shape description algorithms will be used for this purpose and the template matching approach will be applied. Firstly, the algorithms based on the transformation of points from Cartesian to polar coordinates will be investigated, since they obtained very promising results in the problem of shape recognition (e.g. [14, [15]).

Acknowledgments. The author of this paper wishes to thank gratefully MSc W. Batniczak for significant help in developing and exploring the presented approach.

\section{References}

1. Chandaria, J., Thomas, G., Bartczak, B., Koeser, K., et al.: Real-Time Camera Tracking in the MATRIS Project. In: Proc. of the International Broadcasting Convention, IBC, Amsterdam, the Netherlands, September 7-11 (2006)

2. TRACAB homepage, http://www.tracab.com

3. Papaioannou, E., Karpouzis, K., De Cuetos, P., Karagiannis, V., et al.: MELISA A Distributed Multimedia System for Multi-Platform Interactive Sports Content Broadcasting. In: Proc. of the 30th EUROMICRO Conference, Rennes, France, September 1-3 (2004)

4. Andrade, E.L., Khan, E., Woods, J.C., Ghanbari, M.: Player Identification in Iinteractive Sport Scenes Using Region Space Analysis, Prior Information and Number recognition. In: Proc. of the International Conference on Visual Information Engineering, pp. 57-60 (July 2003)

5. Ghanbari, M., Andrade, E.L., Woods, J.C.: Outlier Removal for Player Identification in Interactive Sport Scenes Using Region Analysis. In: Proc. of the International Workshop on Image Analysis for Multimedia Interactive Services, WIAMIS 2004, Lisbon, Portugal (2004)

6. Frejlichowski, D., Wierzba, P.: A Face-Based Automatic Identification of Football Players During a Sport Television Broadcast. Polish Journal of Environmental Studies 17(4C), 406-409 (2008)

7. Kukharev, G., Kuzminski, A.: Biometric Techniques. Part 1. In: Face Recognition Methods (in Polish). WIPS Press, Szczecin (2003)

8. Yow, D., Yeo, B.-L., Yeung, M., Liu, B.: Analysis and presentation of Soccer highlights from digital video. In: Li, S., Teoh, E.-K., Mital, D., Wang, H. (eds.) ACCV 1995. LNCS, vol. 1035, pp. 499-503. Springer, Heidelberg (1996)

9. Choi, S., Seo, Y., Kim, H., Hong, K.-S.: Where Are the Ball and Players?: Soccer Game Analysis with Color-based Tracking and Image Mosaick. In: Del Bimbo, A. (ed.) ICIAP 1997. LNCS, vol. 1311, pp. 196-203. Springer, Heidelberg (1997)

10. Leonardi, R., Migliorati, P.: Semantic Indexing of Multimedia Documents. Trans. on IEEE Multimedia 9(2), 44-51 (2002)

11. Assfalg, J., Bertini, M., Colombo, C., del Bimbo, A., Nunziati, W.: Semantic Annotation of Soccer Videos: Automatic Highlights Identification. Computer Vision and Image Understanding 92, 285-305 (2003) 
12. Ancona, N., Cicirelli, G., Stella, E., Distante, A.: Ball Detection in Static Images with Support Vector Machines for Classification. Image and Vision Computing 21, 675-692 (2003)

13. Frejlichowski, D.: Image Segmentation for the Needs of the Automatic Identification of Players Basing on Kits During the Sport Television Broadcast (in Polish). Methods of Applied Computer Science (2), 45-54 (2007)

14. Frejlichowski, D.: An Algorithm for Binary Contour Objects Representation and Recognition. In: Campilho, A., Kamel, M.S. (eds.) ICIAR 2008. LNCS, vol. 5112, pp. 537-546. Springer, Heidelberg (2008)

15. Frejlichowski, D.: Analysis of Four Polar Shape Descriptors Properties in an Exemplary Application. In: Bolc, L., Tadeusiewicz, R., Chmielewski, L.J., Wojciechowski, K. (eds.) ICCVG 2010, Part I. LNCS, vol. 6374, pp. 376-383. Springer, Heidelberg (2010) 